\title{
A Comparative Buckling Analysis of Silicon Carbide Nanotube and Boron Nitride Nanotube
}

\author{
Kadir Mercan \\ Akdeniz University, Civil Engineering Department, Antalya-TURKIYE \\ E-mail address: mercankadir@akdeniz.edu.tr \\ Received date: December 2016 \\ Accepted date: December 2016
}

\begin{abstract}
The popularity of nanodevices is gaining a vital importance nowadays. These supersmall sized devices started to be used in human body as in computers. The first using of medical nanotechnology is to deliver of medications with the hope that 'magic bullet' chemotherapy to eradicate tumor cells with lower systemic toxicity. Carbon nanotubes are widely used in nanotechnology and many works have been done about it. With the science always need better materials with better properties, scientist have developed Carbon nanotubes to Silicon carbide nanotubes. On the other hand, another king of nanotube with better stability properties than Carbon nanotubes is Boron nitride nanotube. In this work, the stability of the Silicon nanotube and Boron nitride nanotubes are investigated and compared in buckling case. The stability of these nanotubes have an important role since it is used in high-tech equipment and started to be implanted inside of human body. In this article, the buckling analysis SiCNT and BNNT is investigated by using Euler-Bernoulli beam theory for different boundary conditions. Results are presented in figures and table.
\end{abstract}

Keywords: Silicon carbide, Boron nitride, nanotube, buckling, Euler-Bernoulli.

\section{Introduction}

Nanometer materials have attracted much interest due to their superior material properties and potential applications in electronic sensor and devices. These electronic sensor and devices have been used in many areas such as aerospace, computers, biotechnology, and optoelectronic. Carbon nanotubes are the first tube form of graphene sheets used in nanoelectronic devices. Because of being the first nanotube, much research has been made about its material properties, stability, and conductivity. The extraordinary mechanical strength, elasticity, and conductivity have made the nanotubes one the most popular research area in past decade. One of the weak sides of carbon nanotube was its durability under very high temperatures. Carbon nanotubes are capable to stay stable up to $600^{\circ} \mathrm{C}$ [1]. In order to use nanotubes in devices-sensors which have to work under very high temperature such aerospace, researchers have developed a new kind of nanotube by combining Carbon and Silica atoms in the graphene sheet. First, the NASA Glenn Research Center has collaborated with Rensselaer and produced Silicon Carbide Nanotubes (SiCNTs) [2]. More recently, Pei [3] have found Silicon Nanotubes which are considered as a kind of self-assembled nanotube which can form crystal structures. Silicon Carbide Nanotubes have attracted much attention due to its better material properties than Carbon nanotubes. SiCNT is capable to stay stable up 
to $1000^{\circ} \mathrm{C}[1]$. The capability of staying stable under higher temperature has made SiCNTs one of the most popular nanotechnological research area by taking over the popularity of CNTs in past few years. On the other hand, another popular kind of nanotube is Boron Nitride Nanotube. The specialty of BNNT is their higher elasticity. The Young modulus of BNNT is $1.8 \mathrm{TPa}$ whereas CNT is $1 \mathrm{TPa}$ and $\mathrm{SiCNT}$ is $0.62 \mathrm{TPa}$ [1]. Many researchers have been studied those nanotubes by using the theory for modeling of nano/micro sized mechanical or biological systems [4-12]. As nanotubes are applied in nano size such as atomic force microscope, nano bridges, carbon nanotubes, nanowires and microelectro mechanical systems (MEMS), nano actuators and sensors. The scale effect such as play an important role in models, many researches have been made in literature in order to show the small-size effect [13-25]. Classical theories cannot be sufficient to calculate the critical buckling loads of nanosized models. To address this issue various kind of size effect theories such as nonlocal elasticity theory, couple stress theory, surface elasticity theory etc. have been used [28-44].

In Fig. 1. A typical Silicon Carbide graphene sheet and a typical Boron Nitride graphene sheet are demonstrated. As it can be seen from the figure, Silicon Carbide graphene sheet is produced from the combine of Carbon atoms (in black) and Silica atoms (in yellow). By rolling the graphene sheet, Silicon Carbide Nanotube can be obtained. The same process is valid for obtain the Boron Nitride nanotube from Boron Nitride graphene sheet.

In previous papers, the buckling analysis of Silicon carbide nanotube [26] and Boron nitride nanotube [27] have been studied. In this work, we aimed to compare the critical buckling forces of both nanotubes.

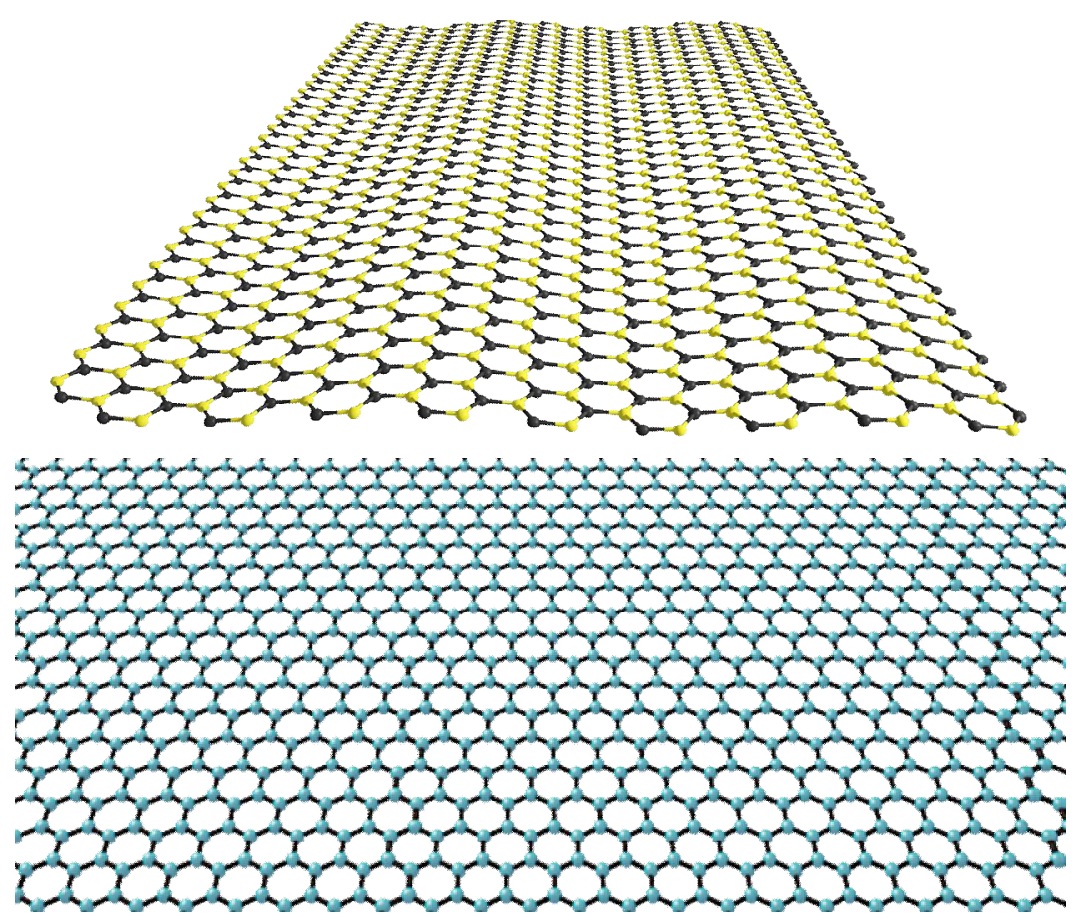

Fig. 1. Demonstration graphene sheets 


\section{Buckling analysis of nanotubes}

The demonstration of silicon carbide nanotube and boron nitride nanotube is shown in Fig.(2). In order to calculate the critical buckling load of the model, Euler-Bernoulli beam theory is used for different boundary conditions. Results are obtained for both BNNT and SiCNT. For modeling, $L$ is the length; $R_{\text {avg }}$ is average radius, $D_{\text {avg }}$ average diameter, $t$ thickness, $E$ Young's modulus of the nanotube.
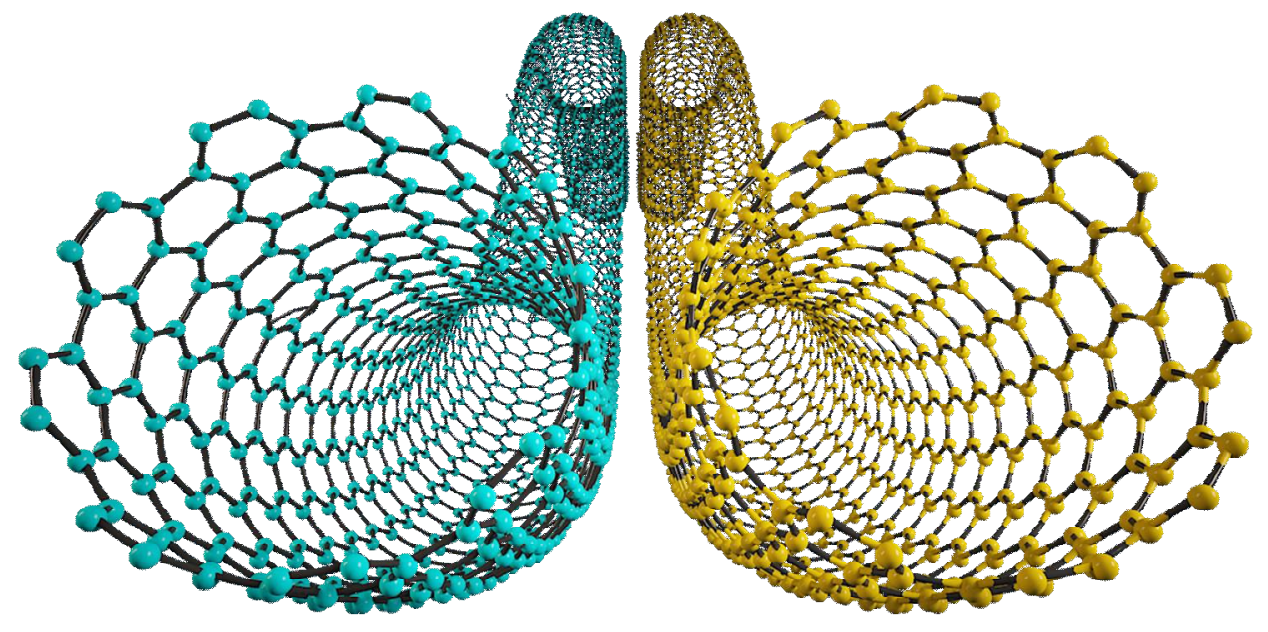

Fig. 2. Demonstration of Boron Nitride Nanotube and Silicon Carbide Nanotube respectively

Silcon carbide nanotubes are tubes which contain both Si and $\mathrm{C}$ atoms bonded each other. In this work, as it can be seen from Fig. 1, the type of which three Si atoms are bonded to one C atoms. Calculations have been made for different types of boundary conditon by employing Euler-Bernoulli classical beam theory. The mechanical continuum model of nanotube is shown in Fig. 3. The length of the nanotube is shown with ' $L$ ', the average radius with ' $R_{\text {avg }}$ ' and the thickness with ' $t$ '. In continuum model, the nanotube will be modeled as a perfect cylindrical shaped tube with a constant inner and outer diameter. The average radius ' $R_{\text {avg' }}$ ' is obtained by using the arithmetical average of the inner and outer radius. The thickness ' $t$ ' is the difference between the inner and outer radius.
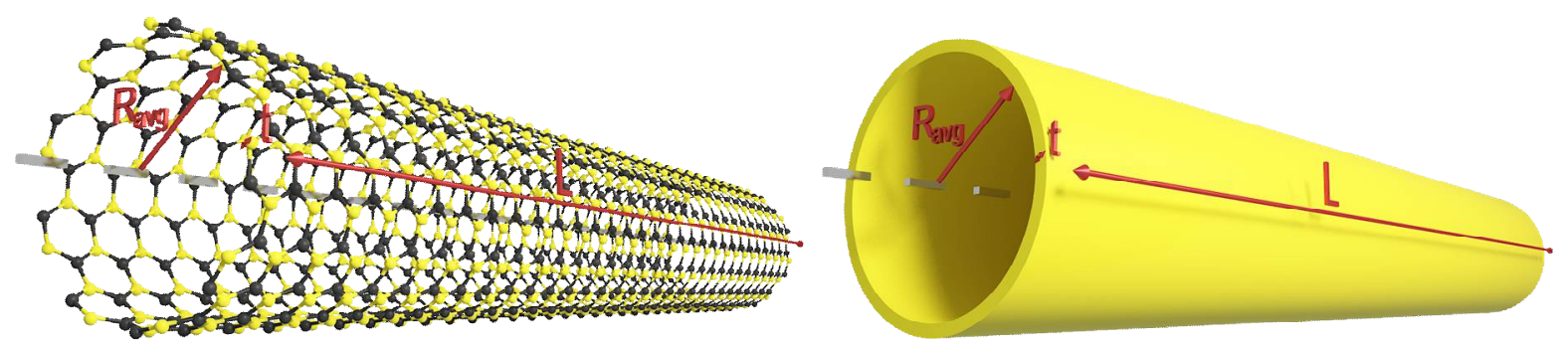

Fig. 3. Real and continuum model nanotube 


\section{Euler-Bernoulli formulation}

The buckling equation of a beam is:

$$
E I \frac{d^{4} y}{d x^{4}}+P \frac{d^{2} y}{d x^{2}}=0
$$

If setting $\alpha^{2}=\frac{P}{E I}$, Eq.(1) can be simplified as:

$$
y^{l v}+\alpha^{2} y^{l l}=0
$$

If setting $y=e^{r x}$ Eq.(2) can be simplified as:

$$
B r^{4} e^{r x}+\alpha^{2} B r^{2} e^{r x}=0
$$

By reducing Eq.(3), we can obtain:

$$
r^{4}+\alpha^{2} r^{2}=0
$$

Solving Eq.(4), the result is:

$$
\begin{gathered}
r^{2}=-\alpha^{2} \\
r_{1,2}=0 \quad \text { and } \quad r_{3,4}= \pm i \alpha
\end{gathered}
$$

$r_{1,2}$ and $r_{3,4}$ are two pairs of single complex root of Eq.(4).

By substitution roots into Eq.(5) and solving it, we obtain:

$$
y=C_{1} \sin \alpha x+C_{2} \cos \alpha x+C_{3} x+C_{4}
$$

$C_{1}, C_{2}, C_{3}, C_{4}$ are constants which can be obtained from boundary conditions. The first order derivative of Eq.(6) is:

$$
y^{\prime}=C_{1} \alpha \cos \alpha x-C_{2} \alpha \sin \alpha x+C_{3}
$$

The second order derivative of Eq.(6) is:

$$
y^{\prime \prime}=-C_{1} \alpha^{2} \sin \alpha x-C_{2} \alpha^{2} \cos \alpha x
$$

The third order derivative of Eq.(6) is:

$$
y^{\prime \prime \prime}=-C_{1} \alpha^{3} \cos \alpha x+C_{2} \alpha^{3} \sin \alpha x
$$
followed:

For a beam which is Clamped-Free supported, the boundary conditions would be as followed: 


$$
y(0)=y^{\prime}(0)=0, \quad y^{\prime \prime}(l)=y^{\prime \prime \prime}(l)+\alpha^{2} y^{\prime}(l)=0
$$

By substituting boundary conditions into Eq.(6), Eq.(7), Eq.(8) and Eq.(9) we obtain:

$$
\begin{gathered}
y(0)=C_{2}+C_{4}=0 \\
y^{\prime}(0)=C_{1} \alpha+C_{3}=0 \\
y^{\prime \prime}(l)=-C_{1} \alpha^{2} \sin \alpha l-C_{2} \alpha^{2} \cos \alpha l=0 \\
y^{\prime \prime \prime}(l)+\alpha^{2} y^{\prime}(l)=C_{3} \alpha^{2}=0
\end{gathered}
$$

As it is mentioned above $C_{1}, C_{2}, C_{3}, C_{4}$ are constants and we can obtain those constant by using Eq.(11), Eq.(12), Eq.(13) and Eq.(14). The solution is obtained as follow:

$$
\alpha^{5} \cos (\alpha l)=0
$$

There are 2 possibilities which make the Eq.(15) equal to zero.

$$
\begin{aligned}
& \alpha^{5}=0 \\
& \cos (\alpha l)=0
\end{aligned}
$$

By substituting $\alpha^{2}=\frac{P}{E I}$ into Eq.(17) we can obtain:

$$
\cos \left(\sqrt{\frac{P}{E I}} l\right)=0, \text { so } \sqrt{\frac{P}{E I}} l=n \frac{\pi}{2}
$$

So the buckling load can be obtained via this formula:

$$
P=\frac{n^{2} \pi^{2} E I}{4 l^{2}}
$$

The solutions are similarly obtained for other types of boundary conditions.

\subsection{Numerical examples}

In this study, the buckling of SiCNTs and BNNTs with various boundary conditions is investigated via classical Euler-Bernoulli beam theory. Some of the results which are showing the critical buckling loads for Clamped-Free, Simple-Simple, Clamped-Simple, ClampedClamped boundary conditions are in Figure 4.The elasticity modulus of SiCNT is E=0.62 $\mathrm{TPa}$, the elasticity modulus of BNNT is $\mathrm{E}=1.8 \mathrm{TPa}[1]$, the thickness is $\mathrm{t}=0.075 \mathrm{~nm}$ for both nanotubes, and the moment of inertia is obtained as $\mathrm{I}=\pi \mathrm{t} \mathrm{R}_{\mathrm{avg}}{ }^{3} \cdot\left(\mathrm{R}_{\mathrm{avg}}=\mathrm{D}_{\mathrm{avg}} / 2\right)$. As it can be seen in Figure 4, the buckling load is investigated for simply supported, clamped, propped and cantilever boundary conditions respectively. 


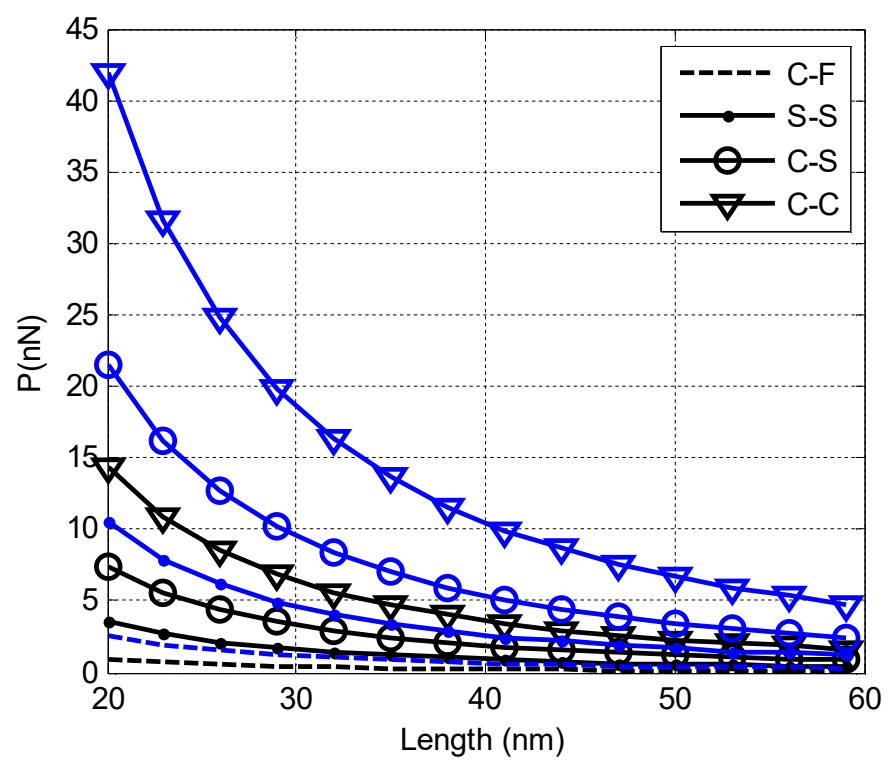

Fig.4. Variation of buckling load of SiCNT (in black) and BNNT (in blue) with different boundary conditions.

In Fig. 4 blue line represent the critical buckling load of BNNT, black lines represent the critical buckling load of SiCNT for C-F, S-S, C-S, C-C boundary conditions. As it can be seen from the figure the buckling load is decreasing dramatically with the increasing of length for both nanotubes.

\section{Concluding remarks}

Buckling analysis of silicon carbide nanotube (SiCNT) and boron nitride nanotube (BNNT) is investigated for various boundary conditions (C-F, S-S, C-S, C-C). Present equations from literature are used in order to calculate the critical buckling loads. Results are presented in a figure.

\section{Acknowledgements}

The financial support of the Scientific Research Projects Unit of Akdeniz University is gratefully acknowledged.

\section{References}

[1] Schulz, M., Shanov, V., Yin, Z., Nanotube Superfiber Materials: Changing Engineering Design2013; William Andrew,2013.

[2] Lienhard, M.A., Larkin, D.J., Silicon Carbide Nanotube Synthesized. 2003.

[3] Pei, L., Tang, Y., Chen, Y., Guo, C., Li, X., Yuan, Y., Zhang, Y., Preparation of silicon carbide nanotubes by hydrothermal method. Journal of applied physics, 99(11), 114306, 2006. 
[4] Wang, Q., Liew, K., Application of nonlocal continuum mechanics to static analysis of micro-and nano-structures. Physics Letters A, 363(3), 236-242, 2007.

[5] Pradhan, S., Phadikar, J., Small scale effect on vibration of embedded multilayered graphene sheets based on nonlocal continuum models. Physics letters A, 373(11), 1062-1069, 2009.

[6] Murmu, T., Pradhan, S., Small-scale effect on the vibration of nonuniform nanocantilever based on nonlocal elasticity theory. Physica E: Low-dimensional Systems and Nanostructures, 41(8), 1451-1456, 2009.

[7] Mercan, K., Civalek, Ö., Demir, Ç., Akgöz, B. Buckling of boron nitride nanotubes surrounded by an elastic matrix.International Conference on Mechanics of Composites, Year.

[8] Hu, Y.-G., Liew, K.M., Wang, Q., He, X., Yakobson, B., Nonlocal shell model for elastic wave propagation in single-and double-walled carbon nanotubes. Journal of the Mechanics and Physics of Solids, 56(12), 3475-3485, 2008.

[9] Demir, Ç., Civalek, Ö., Akgöz, B., Free vibration analysis of carbon nanotubes based on shear deformable beam theory by discrete singular convolution technique. Mathematical and Computational applications, 15(1), 57-65, 2010.

[10] Civalek, Ö., Akgöz, B., Vibration analysis of micro-scaled sector shaped graphene surrounded by an elastic matrix. Computational Materials Science, 77, 295-303, 2013.

[11] Akgöz, B., Civalek, Ö., Mechanical analysis of isolated microtubules based on a higher-order shear deformation beam theory. Composite Structures, 118, 9-18, 2014.

[12] Akgöz, B., Civalek, Ö., Buckling analysis of cantilever carbon nanotubes using the strain gradient elasticity and modified couple stress theories. Journal of Computational and Theoretical Nanoscience, 8(9), 1821-1827, 2011.

[13] Ajori, S., Ansari, R., Torsional buckling behavior of boron-nitride nanotubes using molecular dynamics simulations. Current Applied Physics, 14(8), 1072-1077, 2014.

[14] Akgöz, B., Civalek, Ö., Strain gradient elasticity and modified couple stress models for buckling analysis of axially loaded micro-scaled beams. International Journal of Engineering Science, 49(11), 1268-1280, 2011.

[15] Akgöz, B., Civalek, Ö., A new trigonometric beam model for buckling of strain gradient microbeams. International Journal of Mechanical Sciences, 81, 88-94, 2014.

[16] Ansari, R., Ajori, S., Molecular dynamics study of the torsional vibration characteristics of boron-nitride nanotubes. Physics Letters A, 378(38), 2876-2880, 2014.

[17] Arani, A.G., Jamali, S.A., Amir, S., Maboudi, M.J., Electro-thermo--mechanical nonlinear buckling of Pasternak coupled DWBNNTs based on nonlocal piezoelasticity theory. Turkish Journal of Engineering and Environmental Sciences, 37(3), 231-246, 2014.

[18] Arani, A.G., Roudbari, M., Nonlocal piezoelastic surface effect on the vibration of visco-Pasternak coupled boron nitride nanotube system under a moving nanoparticle. Thin Solid Films, 542, 232-241, 2013.

[19] Aydogdu, M., Axial vibration of the nanorods with the nonlocal continuum rod model. Physica E: Low-dimensional Systems and Nanostructures, 41(5), 861-864, 2009.

[20] Ece, M., Aydogdu, M., Nonlocal elasticity effect on vibration of in-plane loaded double-walled carbon nano-tubes. Acta Mechanica, 190(1-4), 185-195, 2007.

[21] Filiz, S., Aydogdu, M., Axial vibration of carbon nanotube heterojunctions using nonlocal elasticity. Computational Materials Science, 49(3), 619-627, 2010.

[22] Jamalpoor, A., Hosseini, M., Biaxial buckling analysis of double-orthotropic microplate-systems including in-plane magnetic field based on strain gradient theory. Composites Part B: Engineering, 75, 53-64, 2015. 
[23] Xie, G., Han, X., Liu, G., Long, S., Effect of small size-scale on the radial buckling pressure of a simply supported multi-walled carbon nanotube. Smart materials and structures, 15(4), 1143, 2006.

[24] Panchal, M.B., Upadhyay, S., Cantilevered single walled boron nitride nanotube based nanomechanical resonators of zigzag and armchair forms. Physica E: Lowdimensional Systems and Nanostructures, 50, 73-82, 2013.

[25] Shokuhfar, A., Ebrahimi-Nejad, S., Effects of structural defects on the compressive buckling of boron nitride nanotubes. Physica E: Low-dimensional Systems and Nanostructures, 48, 53-60, 2013.

[26] Mercan, K., Civalek, O., Buckling Analysis of Silicon Carbide Nanotubes (SiCNTs). International Journal of Engineering \& Applied Sciences, 8(2), 101-108, 2016.

[27] Mercan, K., Civalek, O., DSC method for buckling analysis of boron nitride nanotube (BNNT) surrounded by an elastic matrix. Composite Structures, 143, 300-309, 2016.

[28] Baltacioğlu, A., Civalek, Ö., Akgöz, B., Demir, F., Large deflection analysis of laminated composite plates resting on nonlinear elastic foundations by the method of discrete singular convolution. International Journal of Pressure Vessels and Piping, 88(8-9), 290-300, 2011.

[29] Civalek, Ö., Finite Element analysis of plates and shells. Elazı̆̆: Firat University, 1998.

[30] Civalek, Ö., Geometrically non-linear static and dynamic analysis of plates and shells resting on elastic foundation by the method of polynomial differential quadrature (PDQ). 2004, Ph. D. Thesis, Firat University, Elazig, 2004 (in Turkish).

[31] Civalek, Ö., Analysis of thick rectangular plates with symmetric cross-ply laminates based on first-order shear deformation theory. Journal of Composite Materials, 42(26), 2853-2867, 2008.

[32] Civalek, Ö., Akgöz, B., Vibration analysis of micro-scaled sector shaped graphene surrounded by an elastic matrix. Computational Materials Science, 77, 295-303, 2013.

[33] Civalek, Ö., Demir, Ç., and Akgöz, B., Static analysis of single walled carbon nanotubes (SWCNT) based on Eringen's nonlocal elasticity theory. International Journal of Engineering and Applied Sciences, 2(1), 47-56, 2009.

[34] Civalek, Ö., Korkmaz, A., and Demir, Ç., Discrete singular convolution approach for buckling analysis of rectangular Kirchhoff plates subjected to compressive loads on two-opposite edges. Advances in Engineering Software, 41(4), 557-560, 2010.

[35] Demir, Ç., Civalek, Ö. Nonlocal Finite Element Formulation for Vibration. International Journal of Engineering and Applied Sciences, 8, 109-117, 2016.

[36] Civalek, Ö., Demir, Ç. A simple mathematical model of microtubules surrounded by an elastic matrix by nonlocal finite element method. Applied Mathematics and Computation, 289, 335-352, 2016.

[37] Civalek, Ö., Akgöz, B. Vibration analysis of micro-scaled sector shaped graphene surrounded by an elastic matrix. Computational Materials Science, 77, 295-303, 2013.

[38] Civalek, Ö., Demir, Ç., Akgöz, B. Static analysis of single walled carbon nanotubes (SWCNT) based on Eringen's nonlocal elasticity theory. International Journal of Engineering and Applied Sciences, 1, 47-56, 2009.

[39] Akgöz, B., Civalek, Ö. A microstructure-dependent sinusoidal plate model based on the strain gradient elasticity theory. Acta Mechanica, 226, 2277-2294, 2015.

[40] Akgöz, B., Civalek, Ö. Bending analysis of embedded carbon nanotubes resting on an elastic foundation using strain gradient theory. Acta Astronautica, 119, 1-12, 2016.

[41] Akgöz, B., Civalek, Ö. A novel microstructure-dependent shear deformable beam model. Int. J. Mech. Sci., 99, 10-20, 2015. 
[42] Akgöz, B., Civalek, Ö. Buckling analysis of linearly tapered micro-columns based on strain gradient elasticity. Structural Engineering and Mechanics, 48, 195-205, 2013.

[43] Akgöz, B., Civalek, Ö. Bending analysis of FG microbeams resting on Winkler elastic foundation via strain gradient elasticity. Composite Structures, 134, 294-301, 2015.

[44] Baltacioğlu, A.K., Akgöz, B., Civalek, Ö. Nonlinear static response of laminated composite plates by discrete singular convolution method. Composite Structures, 93, 153-161, 2010. 